\title{
Thermal stabilization of superconducting sigma strings and their drum vortons
}

\author{
Brandon Carter \\ Observatoire de Paris-Meudon, 92195 Meudon, France \\ Robert H. Brandenberger \\ Physics Department, Brown University, Providence, Rhode Island 02912 \\ and TH Division, CERN, 1211 Geneva 23, Switzerland \\ Anne-Christine Davis \\ DAMTP, Center for Mathematical Sciences, University of Cambridge, Wilberforce Road, Cambridge, CB3 OWA, United Kingdom
}

(Received 17 February 2002; published 14 May 2002)

\begin{abstract}
We discuss various issues related to stabilized embedded strings in a thermal background. In particular, we demonstrate that such strings will generically become superconducting at moderately low temperatures, thus enhancing their stability. We then present a new class of defects-drum vortons-which arise when a small symmetry breaking term is added to the potential. We display these points within the context of the $\mathrm{O}(4)$ sigma model, relevant for hadrodynamics below the QCD scale. This model admits "embedded defects" (topological defect configurations of a simpler-in this case $\mathrm{O}(2)$ symmetric — model obtained by imposing an embedding constraint) that are unstable in the full model at zero temperature, but that can be stabilized (by electromagnetic coupling to photons) in a thermal gas at moderately high termperatures. It is shown here that below the embedded defect stabilization threshold, there will still be stabilized cosmic string defects. However, they will not be of the symmetric embedded vortex type, but of an "asymmetric" vortex type, and are automatically superconducting. In the presence of weak symmetry breaking terms, such as arise naturally when using the $\mathrm{O}$ (4) model for hadrodynamics, the strings become the boundary of a new kind of cosmic sigma membrane, with tension given by the pion mass. The string current would then make it possible for a loop to attain a (classically) stable equilibrium state that differs from an "ordinary" vorton state by the presence of a sigma membrane stretched across it in a drum-like configuration. Such defects will however be entirely destabilized if the symmetry breaking is too strong, as is found to be the case-due to the rather large value of the pion mass - in the hadronic application of the $\mathrm{O}(4)$ sigma model.
\end{abstract}

DOI: 10.1103/PhysRevD.65.103520

PACS number(s): 98.80.Cq

\section{INTRODUCTION}

The purpose of this work is to follow up the work of Nagasawa and Brandenberger [1] who considered the possibility of thermal stabilization, via electromagnetic coupling, of vortex defects, i.e. cosmic strings, in a sigma model characterized by $\mathrm{O}(4)$ symmetry with a set of degenerate vacuum states having the topology of a 3-sphere.

Since the homotopy structure of the 3 -sphere is trivial, such a model does not have stationary vacuum defects of a topologically stable kind. However, this model (involving charged and neutral pion fields as well as the sigma field) contains a subset of solutions that is identifiable as the complete set of solutions of an "embedded" model (involving just the neutral pion and the sigma field) characterized by $\mathrm{O}(2)$ symmetry. This embedded model has a set of degenerate vacuum solutions having the topology of a circle, and therefore admits stationary vacuum vortex defects of a topologically stable kind, which were called pion strings in the initial paper [2] on this subject. ${ }^{1}$ These stationary topological

\footnotetext{
${ }^{1}$ In this paper we will restrict our attention to the classical sigma model and not touch on the rich variety of defects which can exist when the quantum nature of QCD (in particular at high baryon density) is taken into account (see e.g. [3-9] for discussions of such defects).
}

defect configurations of the embedded $\mathrm{O}(2)$ model constitute what are known [10-13] as embedded defects within the framework of the full model, but as their energy is not minimized in the broader framework of the full $\mathrm{O}(4)$ model they will not be stable in this more general context.

The point made by Nagasawa and Brandenberger [1] was that the background reference states that are relevant in cosmological contexts are commonly not vacuum states but thermal equilibrium states, for which topological defects of the embedded $\mathrm{O}(2)$ model can be stable as vortex defects of the full model. The possibility of creating such vortex defects, i.e. cosmic strings, arises from breaking of the $\mathrm{O}(4)$ symmetry by thermal effects mediated by electromagnetic coupling. Such stabilization of an embedded defect [i.e. of a topological defect of the embedded $\mathrm{O}(2)$ symmetric model] does however require that the product of the relevant electric coupling constant $e$ and the temperature $\Theta$ should be sufficiently large.

The first thing we wish to point out here is that topologically stabilized vortex defects of thermal (not vacuum) equilibrium states will exist for any non-zero value of the product $e \Theta$, even if it is very small (as long as the temperature is higher than the temperature of recombination, below which the thermal analysis used in this paper breaks down). For large values of $e \Theta$ these topological defects include the embedded defects referred to above. However, for smaller values of $e \Theta$ the topological defects are not configurations of 
the embedded model, but are of a mathematically less trivial kind with the important property that (unlike their embedded counterparts at higher temperature) they are automatically "superconducting" in the sense of Witten [14]. As first observed by Davis and Shellard [15], such a conductivity property allows cosmic string loops to form vortons, i.e. centrifugally supported equilibrium states, which under a wide range of conditions will actually be stable [16].

The foregoing considerations are based on the supposition that the underlying field model has non-thermal vacuum states characterized by strict $\mathrm{O}(4)$ symmetry, with respect to which the pions are identifiable as Goldstone bosons which as such must have zero mass. However, for a more realistic description, allowing for a finite pion mass that is actually observed, the Lagrangian of the model has to be augmented by the inclusion of a small intrinsic $\mathrm{O}(4)$ symmetry breaking term. This removes the degeneracy of the vacuum, as well as of the thermal equilibrium states, so there is no longer any possibility of forming a topologically stable defect, whether of the vacuum or of a thermal equilibrium state at finite temperature.

There is however the possibility at finite temperature of setting up a stationary state of a more interesting kind. One of the purposes of this article is to consider the construction in such a context of a more general kind of (dynamically but not topologically) stable equilibrium configuration that may be described as "drum vorton" (or "frisbee") consisting of a vorton-xlike loop forming the boundary of a drum-type membrane.

It is shown that the existence of such stabilized defects is only possible if the symmetry breaking term is sufficiently small. This condition may be satisfied in other applications, but it is found that it does not hold in the case when the $\mathrm{O}(4)$ sigma model is applied in the hadrodynamic context for which it was originally designed. The failure of the stabilization mechanism in this particular case is attributable to the rather large value of the (destabilizing) pion mass $m_{\pi}$ in conjunction with the rather small value $e^{2} \simeq 1 / 137$ of the (stabilizing) electromagnetic coupling constant.

\section{BOSONIC SIGMA MODEL}

The following work will be based on the use of a sigma model of the usual kind constituting the bosonic sector of the Schweber-Gell-Mann-Levy-type [17,18] hadrodynamic field theory as presented in the recent treatise of Walecka [19]. Such a sigma model is given by a Lagrangian density of the form

$$
\mathcal{L}=\frac{1}{16 \pi} F^{\nu \mu} F_{\mu \nu}-\frac{1}{2}\left(\chi_{\mid \mu}^{*} \chi^{\mid \mu}+\phi_{; \mu}^{*} \phi^{; \mu}\right)-V,
$$

with

$$
V=\frac{\lambda}{4}\left(\chi^{*} \chi+\phi^{*} \phi-\eta^{2}\right)^{2}-\varepsilon \lambda \sigma,
$$

where

$$
F_{\mu \nu}=A_{\nu ; \mu}-A_{\mu ; \nu}, \quad \chi_{\mid \mu}=\chi_{; \mu}+i e A_{\mu} \chi,
$$

in which a semicolon denotes Riemannian covariant differentiation with respect to the spacetime metric $g_{\mu \nu}$ which we take to have signature $(-,+,+,+)$, while the asterisk $(*)$ denotes complex conjugation. In addition to the electromagnetic gauge potential $A_{\mu}$, the independent bosonic fields are a set of four real scalar fields consisting of a pion triplet $\pi_{0}$, $\pi_{1}, \pi_{2}$ and a singlet, $\sigma=\pi_{3}$ say, that combine as the complex (respectively charge coupled and neutral) combinations

$$
\chi=\pi_{1}+i \pi_{2}, \quad \phi=\pi_{3}+i \pi_{0},
$$

so that

$$
\sigma=\frac{1}{2}(\phi+* \phi) .
$$

The other quantities involved are constants, of which $\lambda$ and the usual charge coupling constant $e$ are dimensionless (and respectively large and small, but only moderately, compared with unity) while $\eta$ has the dimensions of a mass (with a value about half that of the pion) and finally $\varepsilon$ has the dimensions of the cube of a mass (that is small compared with that of the pion).

It is evident that [independently of the local U(1) gauge symmetry of the electromagnetic part] there will be a global $\mathrm{O}(4)$ symmetry that will act on the sigma pion sector in the limit when the constants $e$ and $\varepsilon$ are set to zero. This can be seen from the fact that the corresponding limit for the potential has the obviously $\mathrm{O}(4)$ invariant form

$$
V \sim \frac{\lambda}{4}\left(\pi_{0}^{2}+\pi_{1}^{2}+\pi_{2}^{2}+\pi_{3}^{2}-\eta^{2}\right)^{2}
$$

(and similarly for the kinetic term) as $e \rightarrow 0$ and $\varepsilon \rightarrow 0$.

The presence of the symmetry breaking term proportional to $\varepsilon$ is empirically needed [19] to account for the finite (observed) value of the pion mass $m_{\pi}$ that is given by the vacuum state value of the partial derivative

$$
\frac{\partial V}{\partial\left(\chi^{*} \chi\right)}=\frac{\lambda}{2}\left(\chi^{*} \chi+\phi * \phi-\eta^{2}\right) .
$$

The vacuum, i.e. the state for which $V$ is minimum, is evidently characterized by the vanishing of the triplet $\pi_{0}, \pi_{1}$, $\pi_{2}$ while the value of $\sigma$ will be given by the larger, $\sigma_{+}$say, of the pair, $\sigma_{+}, \sigma_{-}$of values where (for vanishing pion fields) $V$ has a local minimum. These restricted minima are given by the highest and lowest solutions of the cubic equation

$$
\sigma_{ \pm}\left(\sigma_{ \pm}^{2}-\eta^{2}\right)=\varepsilon .
$$

The relevant values will be given, for small values of $\varepsilon$, by the expansion

$$
\sigma_{ \pm}= \pm \eta+\frac{\varepsilon}{2 \eta^{2}} \mp \frac{3 \varepsilon^{2}}{8 \eta^{5}}+\mathcal{O}\left\{\varepsilon^{3}\right\} .
$$

Substituting the vacuum state value $\sigma=\sigma_{+}$in the formula 


$$
m_{\pi}^{2}=\frac{2 \partial V}{\partial\left(\chi^{*} \chi\right)}
$$

one finds that for low values of $\varepsilon$ the result will be given by

$$
m_{\pi}^{2}=\frac{\varepsilon \lambda}{\eta}+\mathcal{O}\left\{\varepsilon^{2}\right\}
$$

\section{III. (NEUTRAL) EMBEDDED MODEL}

The configuration space of the preceding model evidently includes an embedded subspace characterized by

$$
\chi=0 \Leftrightarrow \pi_{1}=\pi_{2}=0
$$

which characterizes an (electromagnetically decoupled) "embedded model" with Lagrangian

$$
\mathcal{L}_{\{0\}}=\frac{1}{16 \pi} F^{\nu \mu} F_{\mu \nu}-\frac{1}{2} \phi_{; \mu} * \phi^{; \mu}-V_{\{0\}}
$$

for

$$
V_{\{0\}}=\frac{\lambda}{4}\left(\phi * \phi-\eta^{2}\right)^{2}-\varepsilon \lambda \sigma,
$$

whose solutions will all automatically satisfy the field equations of the complete model (1).

This embedded model does not involve the charge coupling constant (whose actual physical value would be given by $e^{2} \simeq 1 / 137$ ) but it does involve the other symmetry breaking parameter $\varepsilon$. However, in the limit when the latter is set to zero the reduced model will be subject to a global $\mathrm{O}(2)$ symmetry action, as can be seen from the fact that the corresponding limit for the potential has the obviously $\mathrm{O}(2)$ invariant form

$$
V_{\{0\}} \sim \frac{\lambda}{4}\left(\pi_{0}^{2}+\pi_{3}^{2}-\eta^{2}\right)^{2}
$$

(and similarly for the kinetic term) for $\varepsilon \rightarrow 0$. In this limit there will be a set of vacuum states with circular topology characterized by $\phi^{*} \phi=\eta^{2}$. One of the features of this embedded model in the limit of vanishing $\varepsilon$ will therefore be the presence of topological vortex (i.e., string type) defects of the vacuum. Such a configuration will be what is describable as an embedded defect from the point of view of the complete theory whose field equations it will also satisfy in the relevant limit of vanishing $\varepsilon$, but in this broader framework it will be unstable since there will be no topological impediment to its decay via the excitation of the $\chi$ (charged pion) degrees of freedom.

\section{THERMALLY MODIFIED MODELS: GENERAL CONSIDERATIONS}

Field models such as those described above can be modified so as to allow for the effect of a thermal background, with temperature $\Theta$, by replacing the relevant potential function, $\mathcal{V}$, by an appropriately modified function, $\mathcal{V}_{\Theta}$. If the independent field components involved are denoted by $\varphi_{i}$, for some index $i$ labeling the relevant degrees of freedom, then one would expect the effect of small short wavelength fluctuations $\delta \phi_{i}$ to be approximately describable by an expansion of the form

$$
\mathcal{V}_{\Theta}=\mathcal{V}+\frac{\partial \mathcal{V}}{\partial \varphi_{i}}\left\langle\delta \varphi_{i}\right\rangle+\frac{1}{2} \frac{\partial^{2} \mathcal{V}}{\partial \varphi_{i} \partial \varphi_{j}}\left\langle\delta \varphi_{i} \delta \varphi_{j}\right\rangle+\cdots
$$

where the angular brackets denote thermal averages. One would expect the odd power averages to cancel out, starting with the linear contributions

$$
\left\langle\delta \varphi_{i}\right\rangle=0
$$

so the leading contribution will be of quadratic order. One would expect the short wavelength bosonic fluctuations to behave like a simple Bose-Einstein radiation gas for whichusing a formula of Dolan and Jackiw [20], for which a simpler derivation will be provided below in Appendix A-the quadratic contribution will be given simply by

$$
\left\langle\delta \varphi_{i} \delta \varphi_{j}\right\rangle=\frac{\Theta^{2}}{12} \delta_{i j}
$$

Thus under conditions such that (in order for the use of such a thermal potential to be meaningful at all) the background variation length scale is large compared with the thermal length scale-i.e. the magnitude of the thermal symmetry 4 -vector $\beta^{\mu}$ with components $\left\{\Theta^{-1}, 0,0,0\right\}$ in the thermal rest frame-but such that the temperature is small enough (i.e. $\beta^{\mu}$ is large enough) for the higher order terms in Eq. (16) to be neglected, one is led to the use of an approximation given by the formula

$$
\mathcal{V}_{\Theta}-\mathcal{V}=\frac{\Theta^{2}}{24} \delta_{i j} \frac{\partial^{2} \mathcal{V}}{\partial \varphi_{i} \partial \varphi_{j}}
$$

\section{THERMALLY MODIFIED SIGMA MODELS}

In the particular case of the embedded model characterized by Eq. (13) it suffices to identify the components $\varphi$ with the real and imaginary parts of the complex vector $\phi$, and to take $\mathcal{V}$ to be $V_{\{0\}}$, so that the foregoing prescription leads directly to the formula

$$
V_{\{0\}_{\Theta}}-V_{\{0\}}=\Theta^{2} \frac{\lambda}{6}\left(\phi * \phi-\frac{1}{2} \eta^{2}\right) .
$$

It is to be remarked that this adjustment (in which the final term proportional to $\eta$ is dynamically irrelevant because it does not depend on $\phi$ ) is such as to preserve the $\mathrm{O}(2)$ symmetry in the limit $\varepsilon \rightarrow 0$ : it is therefore qualitatively uninteresting for moderate values of $\Theta$, though for higher values (above a critical value $\Theta_{c}$ ) it will have the physically significant effect of removing the degeneracy (and the consequent spontaneous symmetry breaking) of the ground state.

The situation is more complicated for the full sigma model characterized by Eq. (1), because in addition to the potential $V$ given by Eq. (2) there will be another coupling 
energy contribution-coming from the gauge coupling in the kinetic term-so that for the purpose of applying the prescription (19) we need to make the identification

$$
\mathcal{V}=V+\frac{1}{2} e^{2} \chi^{*} \chi A_{\mu} A^{\mu}
$$

In view of the gauge invariance, the four components of the covector $A_{\mu}$ should not all be considered to be dynamically independent. Imposing the thermal gauge condition $\beta^{\mu} A_{\mu}$ $=0$ (which is compatible with the usual Lorentz condition $A_{\mu}^{; \mu}=0$ ) one is left with three independent components given with respect to the thermal rest frame by $A_{1}, A_{2}, A_{3}$. In order to be able to apply the formula (18) on which the prescription (19) is based, the corresponding field components $\varphi_{i}$ must be specified with the appropriate normalization, which can be achieved by taking $\varphi_{i}=A_{i} / \sqrt{4 \pi}$ ( $i$ $=1,2,3$ ) in order for the (unrationalized) kinetic term $F_{\mu \nu} F^{\mu \nu} / 16 \pi$ to reduce (subject to the usual Lorentz gauge condition) to the standard form $\frac{1}{2}\left(\varphi_{1 ; \mu} \varphi_{1}^{; \mu}+\varphi_{2 ; \mu} \varphi_{2}^{; \mu}\right.$ $\left.+\varphi_{3 ; \mu} \varphi_{3}^{; \mu}\right)$. The corresponding expression for the electromagnetic potential will therefore be given by

$$
\mathcal{V}-V=2 \pi e^{2} \chi^{*} \chi\left(\varphi_{1}^{2}+\varphi_{2}^{2}+\varphi_{3}^{2}\right)
$$

It can thus be seen that the corresponding thermally modified version of the complete sigma model (1) will be given by

$$
\mathcal{L}_{\Theta}=\frac{1}{16 \pi} F^{\nu \mu} F_{\mu \nu}-\frac{1}{2}\left(\chi_{\mid \mu}^{*} \chi^{\mid \mu}+\phi_{; \mu}^{*} \phi^{; \mu}\right)-V_{\Theta},
$$

with

$$
V_{\Theta}=V+\mathcal{V}_{\Theta}-\mathcal{V}
$$

in which, by application of Eq. (19), the extra thermal contribution can be seen to be given by

$$
\begin{aligned}
V_{\Theta}-V= & \frac{\Theta^{2}}{2} e^{2}\left(\frac{1}{6} A_{\mu} A^{\mu}+\pi \chi^{*} \chi\right) \\
& +\frac{\Theta^{2}}{2} \frac{\lambda}{2}\left(\chi^{*} \chi+\phi^{*} \phi-\frac{2}{3} \eta^{2}\right) .
\end{aligned}
$$

As in the case of the embedded model, the group of terms at the end (i.e. what remains when $e$ is set to zero) is qualitatively uninteresting for moderate values of $\Theta$, though for higher values (above a critical value $\Theta_{c}$ that will be evaluated below) it will have the physically significant effect of removing the degeneracy (and the consequent spontaneous symmetry breaking) of the ground state.

The part proportional to $e^{2}$ is more interesting. The first term breaks the electromagnetic $\mathrm{U}(1)$ gauge invariance, giving an effective mass $m_{\gamma}=\Theta|e| \sqrt{4 \pi / 6}$ to the photon.

The contribution to Eq. (25) that is of greatest interest for our present purpose is the second term, $\pi e^{2} \Theta^{2} \chi^{*} \chi / 2$, which will break the $\mathrm{O}(4)$ symmetry that would otherwise exist in the limit $\varepsilon \rightarrow 0$.

It can be seen from the thermal generalization

$$
\frac{\partial V_{\Theta}}{\partial\left(\chi^{*} \chi\right)}=\pi \frac{\Theta^{2}}{2} e^{2}+\frac{\lambda}{2}\left(\chi^{*} \chi+\phi^{*} \phi+\frac{\Theta^{2}}{2}-\eta^{2}\right),
$$

of Eq. (7), that the charged pion field will have an effective mass, $m_{\chi}$ say, which will be non-vanishing in this thermally modified case, even in the limit for which the symmetry breaking parameter $\varepsilon$ is set to set to zero, when specified in the usual way by the formula

$$
m_{\chi}^{2}=\frac{2 \partial V_{\Theta}}{\partial\left(\chi^{*} \chi\right)}
$$

in the thermal equilibrium state where $V_{\Theta}$ is minimized.

The analogue of Eq. (8) for the values of $\sigma$ at the restricted minima of $V_{\Theta}$ can be seen to have the form

$$
\sigma_{ \pm}\left(\sigma_{ \pm}^{2}-\eta_{\Theta}^{2}\right)=\varepsilon
$$

with

$$
\eta_{\Theta}^{2}=\eta^{2}-\frac{\Theta^{2}}{2} .
$$

The relevant solution will be given by an expansion analogous to Eq. (9) as

$$
\sigma_{ \pm} \simeq \pm \eta_{\Theta}+\frac{\varepsilon}{2 \eta_{\Theta}^{2}}
$$

in the limit when $\varepsilon \ll \eta_{\Theta}^{3}$. This inequality will fail to be satisfied near the critical temperature, i.e. when $\Theta \simeq \sqrt{2} \eta$, in which case the solution to Eq. (28) will have an order of magnitude given simply by

$$
\sigma_{ \pm} \approx \pm \varepsilon^{1 / 3}
$$

Substituting the vacuum state value $\sigma=\sigma_{+}$in Eq. (27), one obtains a formula of the form

$$
m_{\chi}^{2}=\pi e^{2} \Theta^{2}+m_{\pi_{\Theta}}^{2}
$$

in which the first term will remain even when $\varepsilon$ is set to zero. The other term is the square of the effective mass $m_{\pi_{\Theta}}$ of the uncharged pion field $\pi_{0}$, which will be given simply by

$$
m_{\pi_{\Theta}}^{2} \simeq \frac{\varepsilon \lambda}{\eta_{\Theta}}
$$

which works out as

$$
m_{\pi_{\Theta}}^{2} \simeq m_{\pi}^{2}\left(1-\frac{\Theta^{2}}{2 \eta^{2}}\right)^{-1 / 2}
$$

in the limit when $\varepsilon \ll \eta_{\Theta}^{3}$, while for very small values of $\eta_{\Theta}$, i.e. when $\Theta \simeq \sqrt{2} \eta$, it will be given by 


$$
m_{\pi_{\Theta}}^{2} \approx \lambda \varepsilon^{2 / 3} \approx\left(\frac{\lambda \eta^{2}}{m_{\pi}^{2}}\right)^{1 / 3} m_{\pi}^{2}
$$

\section{STABILIZATION OF EMBEDDED DEFECT}

Nagasawa and Brandenberger pointed out [1] that the presence of an $\mathrm{O}(4)$ symmetry breaking term proportional to $\chi^{*} \chi$ can stabilize the embedded string defect introduced in Sec. III. This string can be viewed as an ordinary global vortex defect of the finite temperature sigma model obtained when setting $\chi=0$ after the finite temperature corrections have been included, i.e. in Eq. (23). The corresponding Lagrangian is

$$
\mathcal{L}_{\Theta\{0\}}=\frac{1}{16 \pi} F^{\nu \mu} F_{\mu \nu}-\frac{1}{2} \phi_{; \mu}^{*} \phi^{; \mu}-V_{\Theta\{0\}},
$$

with

$$
V_{\Theta\{0\}}-V_{\{0\}}=\frac{1}{12} \Theta^{2} e^{2} A_{\mu} A^{\mu}+\Theta^{2} \frac{\lambda}{4}\left(\phi^{*} \phi-\frac{2}{3} \eta^{2}\right) .
$$

Note that this embedded submodel of the thermally extended model is different from, and more realistic than, the direct thermal extension of the original embedded submodel (13). It is to be observed that $V_{\Theta\{0\}}$ differs from the quantity $V_{\{0\}_{\Theta}}$ given by Eq. (20) not only by the photon mass term (which could be got rid of by adopting the more restrictive embedding condition to the effect that $A_{\mu}$ should vanish as well as $\chi$, which is possible because $\chi$ is its only source) but also because, unlike $V_{\{0\}_{\Theta}}$, the effective potential $V_{\Theta\{0\}}$ allows for the effect of thermal excitations of the field $\chi$ even though the embedding condition set its mean value to zero. This observation serves as a reminder that a fully realistic treatment would require the inclusion of further terms allowing for the thermal excitation of the fermionic degrees of freedom whose neglect from the outset-on the grounds that we are considering cases where their mean value is zerowas justifiable as a good approximation for the zero temperature limit, but less so at finite temperature.

It can be seen that the potential for this embedded model (36) will be given by an expression of the form

$$
V_{\Theta\{0\}}+\varepsilon \lambda \sigma=\frac{e^{2}}{12} \Theta^{2} A_{\mu} A^{\mu}+\frac{\lambda}{4}\left(\phi * \phi+\frac{\Theta^{2}}{2}-\eta^{2}\right)^{2}+C_{\Theta},
$$

where $C_{\Theta}$ is a temperature dependent contribution that is constant in the sense of being independent of the dynamical field variables, and that is therefore irrelevant in so far as its effect in the Lagrangian is concerned. It is evident that the condition for the degeneracy of the ground state and the existence of the embedded vortex defect in the limit when $\varepsilon$ is set to zero is that the temperature should be less than a critical value given simply by $\eta$, i.e.

$$
\Theta<\Theta_{\mathrm{c}}, \quad \Theta_{\mathrm{c}}=\sqrt{2} \eta .
$$

The positivity property of the quantity $\chi^{*} \chi$ whose vanishing characterizes the embedding under consideration means that the stability of the embedded solution can be checked simply by verifying the positivity of the derivative (26) on the embedding, $\chi=0$, where it reduces simply to

$$
\frac{\partial V_{\Theta}}{\partial\left(\chi^{*} \chi\right)^{*} \chi=0}=\pi \frac{\Theta^{2}}{2} e^{2}+\frac{\lambda}{2}\left(\phi^{*} \phi+\frac{\Theta^{2}}{2}-\eta^{2}\right) .
$$

In order for this to remain positive even at the core of the defect where $\phi^{*} \phi$ goes to zero, it is evidently necessary and sufficient to have

$$
\lambda \eta_{\Theta}^{2}<\pi e^{2} \Theta^{2} .
$$

Combining this with the condition (39) for the defect to exist at all, we see that the necessary and sufficient condition for the existence of stable cosmic strings in the limit when $\varepsilon$ is set to zero is that the dimensionless ratio $\Theta / \eta$ should lie within the finite range

$$
\sqrt{2}\left(1+\frac{2 \pi e^{2}}{\lambda}\right)^{-1 / 2}<\frac{\Theta}{\eta}<\sqrt{2}
$$

a requirement that would be satisfied in a broad range of temperature if the dimensionless ratio $2 \pi e^{2} / \lambda$ were reasonably large, but that is rather restrictive if this ratio is small compared with unity as one expects.

The temperature at which the lower inequality of Eq. (42) is saturated will be denoted by $\Theta_{Q}$. The finite temperature effective potential along in $\phi$ and $\chi$ axes is sketched in Fig. 1 for the various temperature ranges considered.

\section{ASYMMETRIC VORTEX DEFECT}

The instability of the embedded $\chi=0$ vortex defect when the temperature is too low to satisfy Eq. (42), i.e. when

$$
\left(1+\frac{2 \pi e^{2}}{\lambda}\right) \Theta^{2}<2 \eta^{2}
$$

does not mean that there cannot be any stable vortex defect, but merely that there cannot be one that satisfies the $\chi$ reflection symmetry condition. The defects that occur in this case must therefore be of the asymmetric kind that has been recently discussed by Axenides et al. [21-23].

For the full sigma model characterized by Eq. (23) the potential given by Eq. (25) can be seen to be expressible, in a manner analogous to Eq. (38) by

$$
\begin{aligned}
V_{\Theta}+\varepsilon \lambda \sigma= & e^{2} \frac{\Theta^{2}}{2}\left(\frac{1}{6} A_{\mu} A^{\mu}+\pi \chi^{*} \chi\right) \\
& +\frac{\lambda}{4}\left(\chi^{*} \chi+\phi^{*} \phi-\eta_{\Theta}^{2}\right)^{2}+C_{\Theta},
\end{aligned}
$$

in which $\eta_{\Theta}$ is given by Eq. (29).

In the limit when $\varepsilon$ vanishes, it is evident that-if and only if the inequality (39) is satisfied so that $\eta_{\Theta}^{2}$ is positivethis model will have a degenerate $\mathrm{O}(2)$ invariant family of ground states that are the same as those of the embedded 


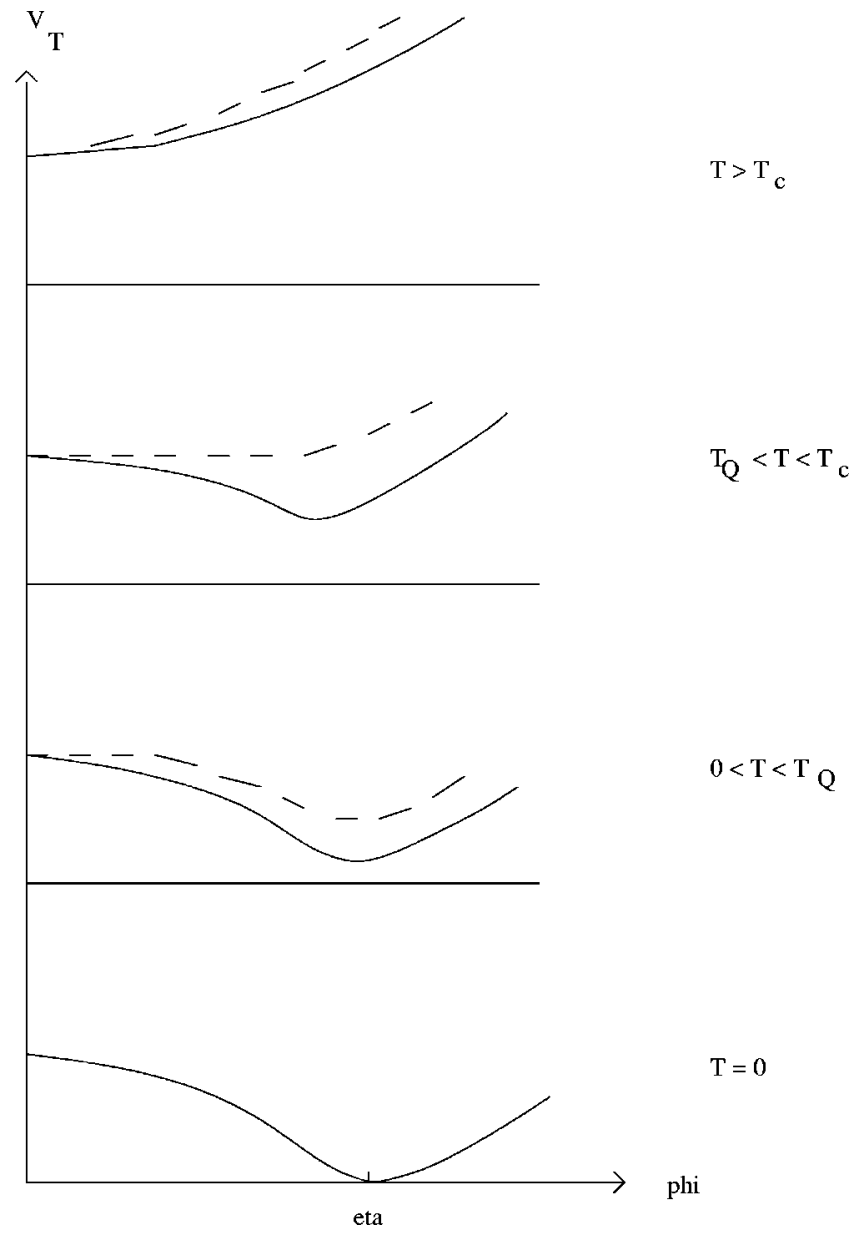

FIG. 1. Sketch of the effective potential $V_{\Theta}$ for the neutral (solid lines) and charged (dashed lines) Higgs fields for different temperature ranges. From bottom to top, the graphs correspond to zero temperature (no stable string), nonzero temperature below the threshold $\Theta_{Q}$ (when a stable conducting string with asymmetric core exists), $\Theta_{Q}<\Theta<\Theta_{c}$ (the temperature range for which the embedded defect with symmetric core is stable), and $\Theta>\Theta_{c}$ (complete symmetry restoration, no string). In the figure the temperature is denoted by $T$ instead of $\Theta$.

model (36), namely the set of states characterized by $A_{\mu} A^{\mu}$ $=0$ and $\chi^{*} \chi=0$ but with $\phi^{*} \phi=\eta_{\Theta}^{2}$.

There must therefore exist corresponding topologically stable vortex defect solutions with a core where $\phi * \phi=0$. When the temperature is in the range (42) these stable topological defects will be identifiable with the embedded defects discussed in the preceding section. However, in the lower temperature range characterized by the inequality (43) [which evidently makes the weaker condition (39) redundant] the topologically stable vortex defects resulting from the potential (44) will no longer satisfy the reflection symmetry condition $\chi=0$. The solution-obtained by minimizing the energy-will presumably be such that $\chi^{*} \chi$ reaches a finite maximum on the core where $\phi^{*} \phi$ vanishes, with a value that will presumably be comparable with, but somewhat less than, the value for which $V_{\Theta}$ is minimized subject to the constraint $\phi=0$, i.e., for the value obtained by solving Eq. (26) with $\phi^{*} \phi$ set to zero, which gives the value of the upper bound as

$$
\chi^{*} \chi=\eta^{2}-\frac{\Theta^{2}}{2}\left(1+\frac{2 \pi e^{2}}{\lambda}\right)
$$

a result that evidently has the necessary property of strict positivity just as long as Eq. (43) is satisfied.

\section{VORTEX CONDUCTIVITY}

The breakdown of the reflection symmetry property $\chi$ $=0$ for vortex defects in the low temperature range given by Eq. (43) implies that (unlike the embedded vortex defects described in Sec. VI) the field configuration in such a vortex will not be uniquely defined but will depend on an arbitrary phase angle, $\varphi$ say, defined as the argument in the expression

$$
\chi=|\chi| \mathrm{e}^{i \varphi}, \quad|\chi|=\sqrt{\chi^{*} \chi}
$$

This means that such a vortex defect will be describable as a superconducting string in the sense of Witten [14], meaning that it will be able to carry a current attributable to slow variation of the phase $\varphi$ along the vortex core. Its properties will therefore depend on the squared magnitude, $\nu^{2}$ say, of the phase gradient, as specified - in such a way that it will be positive for the case of a spacelike gradient with which we shall mainly be concerned here-by

$$
\nu^{2}=\varphi_{; \mu} \varphi^{; \mu} .
$$

In a uniform cylindrically symmetric (Nielsen-OlesenKibble-type) configuration described in terms of corresponding cylindrical time, radial, angular, and longitudinal coordinates, $\{t, \varpi, \theta, z\}$ of the usual kind, physically well-defined quantities will be independent of the time $t$ and the longitudinal coordinate $z$, and will be describable completely as fields on the 2-dimensional orthogonal space sections with circular coordinates $\{\varpi, \theta\}$. In a gauge such that $A_{\mu}$ has no time or longitudinal components the time and longitudinal components of the phase gradient will be physically well defined and therefore uniform, so the phase itself can be taken to be given by an expression of the standard form

$$
\varphi=k z-\omega t
$$

for some constant angular frequency $\omega$ and wave number $k$.

The mechanism described in detail by Peter [24] imposes an upper limit on the admissible value of the longitudinally Lorentz invariant combination $\omega^{2}-k^{2}$. This limit arises from the requirement that the charged condensate field $\chi$ should be effectively confined within a finite length scale, $r_{\chi}$ say, whose order of magnitude can be estimated as being given roughly by

$$
r_{\chi} \approx \frac{1}{\sqrt{m_{\chi}^{2}+k^{2}-\omega^{2}}},
$$

where $m_{\chi}$ is the relevant mass value for the charged pion field, which at zero temperature will be same as that of the uncharged pion field, i.e. $m_{\chi}=m_{\pi}$ with $m_{\pi}$ given by Eq. (11), which evidently satisfies $m_{\pi}^{2} \geqslant \pi e^{2} \Theta^{2}$, so that there will be a charge dependent term that remains even in the limit of 
vanishing $\varepsilon$ and $m_{\pi}$. In terms of this mass value $m_{\chi}$, the confinement condition that is needed for $r_{\chi}$ to remain finite will be given simply by

$$
\omega^{2}-k^{2}<m_{\chi}^{2} .
$$

In such a configuration the original 4-dimensional Lagrangian variational problem reduces to a 2-dimensional energy variation problem for which the original potential function $V_{\Theta}$ has to be replaced by a corresponding 2-dimensional version

$$
V_{\Theta}^{[2]}=V_{\Theta}+\frac{1}{2}\left(k^{2}-\omega^{2}\right) \chi^{*} \chi
$$

to allow for the kinetic contributions from the longitudinal and temporal phase variations. Since the ensuing generalization of Eq. (26) is

$$
\frac{\partial V_{\Theta}^{[2]}}{\partial\left(\chi^{*} \chi\right)}=\pi \frac{\Theta^{2}}{2} e^{2}+\frac{1}{2}\left(k^{2}-\omega^{2}\right)+\frac{\lambda}{2}\left(\chi^{*} \chi+\phi^{*} \phi-\eta_{\Theta}^{2}\right),
$$

this leads to the replacement of Eq. (45) by an upper bound that, for a spacelike current, $k^{2}-\omega^{2}>0$, is more severe, namely

$$
\chi^{*} \chi=\eta^{2}-\frac{\Theta^{2}}{2}\left(1+\frac{2 \pi e^{2}}{\lambda}\right)-\frac{\nu^{2}}{\lambda}
$$

in which the Lorentz invariant quantity

$$
\nu=\sqrt{k^{2}-\omega^{2}}
$$

is identifiable as the phase gradient magnitude that was introduced in Eq. (47).

The necessity that $\chi^{*} \chi$ should be positive implies that - just as a timelike current is subject to the "charge confinement" limit (50) —at the opposite extreme a spacelike current will be subject to a "current quenching" limit of the kind originally discussed by Witten [14], which will be given in the present application by a relation of the form

$$
\nu<\nu_{\mathrm{Q}}
$$

where the quenching limit $\nu_{\mathrm{Q}}$ is given by the formula

$$
\nu_{\mathrm{Q}}^{2}=\lambda \eta_{\Theta}^{2}-\pi e^{2} \Theta^{2},
$$

whose right-hand side itself satisfies a positivity condition that is equivalent to the temperature limit (43). Thus, for fixed temperature $\Theta$, then for $\nu<\nu_{Q}$ the vortices will be stable. An equivalent way to interpret this stability analysis is that for fixed current $\nu$, the vortex will be stable provided $\Theta<\Theta_{\nu}$, where $\Theta_{\nu}$ is given by

$$
\Theta_{\nu}^{2}=2\left(\eta^{2}-\frac{\nu^{2}}{\lambda}\right)\left(1+\frac{2 \pi e^{2}}{\lambda}\right)^{-1}
$$

For $\Theta>\Theta_{\nu}$ the current leaks off the vortex.
In the spacelike current case, for which $k^{2}-\omega^{2}>0$, there will be a locally preferred Lorentz frame characterized by $\omega=0$ and $k=\nu$ in which the total energy per unit length, $U$ say, will exceed the corresponding longitudinal stress magnitude, i.e. the string tension $T$ say, by an amount that can be seen from Eq. (51) to be given by [25]

$$
U-T=\nu^{2} \kappa,
$$

where $\kappa$ is the sectional integral of the condensate amplitude $\chi^{*} \chi$, i.e.,

$$
\kappa=2 \pi \int \chi^{*} \chi r d r
$$

On the basis of Eqs. (49) and (53), the order of magnitude of this sectional integral can be roughly estimated as

$$
\kappa \approx \frac{\lambda \eta_{\Theta}^{2}-\pi e^{2} \Theta^{2}-\nu^{2}}{\lambda\left(m_{\chi}^{2}+\nu^{2}\right)} .
$$

According to the general principles of conducting string theory [25] the total (sectionally integrated) electromagnetic current density $j^{\mu}$ in the string will have a magnitude given in terms of this sectional integral by the formula

$$
j^{\mu} j_{\mu}=e^{2} \mu^{2}, \quad \mu=\kappa \nu,
$$

in which $\mu$ is interpretable as the effective mass per unit phase radian winding number, which can be defined in terms of the relevant equation of state specifying the energy density $U$ as a function of the angular number density $\nu$ by the specification

$$
\mu=\frac{d U}{d \nu}
$$

It can be estimated using Eq. (60) that this effective mass will be given roughly by

$$
\mu \approx \frac{\nu}{\lambda}\left(\frac{m_{\chi}^{2}+\nu_{\mathrm{Q}}^{2}}{m_{\chi}^{2}+\nu^{2}}-1\right)
$$

\section{EQUATION OF STATE}

The value (53) of $\chi^{*} \chi$ is that for which the derivative vanishes, and thus where $V_{\Theta}^{[2]}$ is minimized, subject to the constraint $\phi^{*} \phi=0$. The difference between this minimal value of $V_{\Theta}^{[2]}$ and the thermal equilibrium state value that is its absolute minimum has a value $\Delta V_{\Theta}^{[2]}$ that provides a lower bound on the energy density in the vortex core. This potential energy density difference will be given in the limit of vanishing $\varepsilon$ by the exact formula

$$
\Delta V_{\Theta}^{[2]}=\frac{\pi e^{2} \Theta^{2}+\nu^{2}}{4 \lambda}\left(2 \lambda \eta_{\Theta}^{2}-\nu^{2}-\pi e^{2} \Theta^{2}\right),
$$

which will be valid so long as it does not exceed the upper limit 


$$
\Delta V_{\Theta}^{[2]} \leqslant \frac{\lambda}{4} \eta_{\Theta}^{4}
$$

given by the value of the central energy density in the symmetric "embedded vortex" case that will otherwise be applicable. Thus in consequence of Eq. (55), it can be seen that it will be possible to approximate Eq. (64) by an order of magnitude estimate of the simpler form

$$
\Delta V_{\Theta}^{[2]} \approx \frac{\eta_{\Theta}^{2}}{2}\left(\pi e^{2} \Theta^{2}+\nu^{2}\right) .
$$

This barrier height provides a minimal estimate for the defect energy density on the central axis where $\phi^{*} \phi$ vanishes. The defect core, meaning the region where $\phi^{*} \phi$ differs significantly from its equilibrium state value $\eta_{\Theta}^{2}$, will be characterized by a radial length scale $r_{\phi}$ that can be estimated from the consideration that energy minimization will give rise to a gradient energy density whose order of magnitude $\left(\eta_{\Theta /} r_{\phi}\right)^{2}$ should be comparable with the barrier height $\Delta V_{\Theta}^{[2]}$; i.e., we can expect to have

$$
r_{\phi}^{2} \approx \frac{\eta_{\Theta}^{2}}{2 \Delta V_{\Theta}^{[2]}}
$$

In the case of a $\chi$ reflection symmetric "embedded" defect that will apply when the limit (65) is exceeded, this leads to the simple estimate

$$
r_{\phi} \approx \sqrt{\frac{2}{\eta_{\Theta}}} \sqrt{\lambda}
$$

while in the alternative case of an asymmetric defect, i.e. when the inequality (55) is satisfied, we obtain the estimate

$$
r_{\phi} \approx \frac{1}{\sqrt{\pi e^{2} \Theta^{2}+\nu^{2}}}
$$

It is to be noticed that in order to obtain confinement to a finite core radius it is sufficient but not necessary to have a finite temperature $\Theta$. Even in the zero temperature limit, for which there is no longer any strictly topological stabilization, there can still in principle be a confined defect if there is a non-vanishing spacelike (but not null or timelike) current, i.e., one characterized by a strictly positive value of the quantity $\nu^{2}=k^{2}-\omega^{2}$. However, in practice a defect that depended entirely on this current confining mechanism could not be stable: although compatible with the "quenching" limit (55), the necessary current would have to exceed the more stringent upper limit imposed, as described below, by the requirement of stability with respect to longitudinal perturbations.

It is apparent that in the small $\varepsilon$ limit under consideration in the present section, the core radius (69) will be of the same order of magnitude, $r_{\phi} \approx r_{\chi}$, as the charged condensate confinement radius given by Eq. (49). However, since the kind of string defect we are dealing with is of global rather than local type, its energy density will include an unconfined contribution from the gradient of the $\phi$ field outside the core where $\phi^{*} \phi \simeq \eta_{\Theta}^{2}$. Since this gradient energy density contribution will be given approximately by an expression, namely $\eta_{\Theta}^{2} / 2 r^{2}$, whose radial dependence has the inverse square law character that is typical of a global vortex defect, its integrated contribution for an infinitely long straight string would be logarithmically divergent, so that its effective value in practice will be determined by a long range cut off radius, $R_{\phi}$ say, characterizing the length scale of macroscopic variation of the system. The integrated gradient energy density per unit length of string can thus be estimated to have a magnitude of the order of $\eta_{\Theta}^{2} \ln \left\{R_{\phi} / r_{\phi}\right\}$.

On the presumption that, for large $R_{\phi}$, this gradient contribution will dominate, its value in the limit when the current magnitude $\nu$ vanishes can be used as a matching condition to fix the constant of integration in the solution of Eq. (62) using the estimate (63). We thereby deduce that the equation of state for the string energy density $U$ as a function of the angular winding number density $\nu$ will be given, as a rough approximation, by an expression of the form

$$
U \approx \frac{\eta_{\Theta}^{2}}{2} \ln \left\{R_{\phi}^{2}\left(\nu^{2}+m_{\chi}^{2}\right)\right\}-\frac{\nu^{2}}{2 \lambda},
$$

in the small $\varepsilon$ limit for which the relevant effective mass variable will be given by $m_{\chi}^{2} \approx \pi e^{2} \Theta^{2}$.

On the basis of Eq. (70), the corresponding expression for the string tension $T$ will be given, according to Eqs. (58) and (60), by

$$
T \approx \frac{\eta_{\Theta}^{2}}{2}\left(\ln \left\{R_{\phi}^{2}\left(\nu^{2}+m_{\chi}^{2}\right)\right\}-\frac{2 \nu^{2}}{\nu^{2}+m_{\chi}^{2}}\right)+\frac{\nu^{2}}{2 \lambda} .
$$

It is shown in Appendix B how an appropriately auxiliary field $\Phi$ can be used in a recently formulated procedure [26] for casting such a conducting model into a standard variational form.

\section{LONGITUDINAL STABILITY LIMIT}

The most physically important quantities derivable from the equation of state include the extrinsic (wiggle type) perturbation speed $c_{\mathrm{E}}$ and the longitudinal (sound type) perturbation speed $c_{\mathrm{L}}$, whose squared values are given [25] by the formulas

$$
c_{\mathrm{E}}^{2}=\frac{T}{U}
$$

and [using Eqs. (58), (61) and (62)]

$$
c_{\mathrm{L}}^{2}=-\frac{d T}{d U}=\frac{\nu d \mu}{\mu d \nu},
$$

in which the positivity of the right hand sides is a necessary condition for stability of the short wavength perturbations of the corresponding (wiggle or longitudinal) kind.

The largeness of the logarithmic factor ensures the positivity not just of the energy density $U$ but also of the tension $T$, thus ensuring the satisfaction of the wiggle stability con- 
dition that the quantity in Eq. (72) should be positive throughout the spacelike current range $0 \leqslant \nu^{2}<\nu_{\mathrm{Q}}^{2}$ under consideration for longitudinal stability.

The question of longitudinal stability is less trivial. As in the analogous case [27] of the local string model describing the kind of superconducting vortex obtained [27] from the toy bosonic field model originally proposed by Witten [14], the longitudinal stability requirement

$$
\frac{d \mu}{d \nu}>0
$$

imposes an upper "bunching" stability limit on the physically admissible current amplitude that is more severe that the original "quenching" limit (55) imposed by the requirement $\mu>0$. For Eq. (63), one obtains

$$
\frac{d \mu}{d \nu} \approx \frac{\left(m_{\chi}^{2}+\nu_{\mathrm{Q}}^{2}\right)\left(m_{\chi}^{2}-\nu^{2}\right)}{\lambda\left(m_{\chi}^{2}+\nu^{2}\right)^{2}}-\frac{1}{\lambda} .
$$

Treating the ratio $m_{\chi} \nu_{\mathrm{Q}} /\left(m_{\chi}^{2}+3 \nu_{\mathrm{Q}}^{2}\right)$ as small, which it typically will be (it can never exceed $1 / 3$ ), we see that a necessary and approximately sufficient condition for satisfaction of the longitudinal stability limit (74) is given by the inequality

$$
\frac{\nu^{2}}{\nu_{\mathrm{Q}}^{2}} \lesssim \frac{m_{\chi}^{2}}{3 m_{\chi}^{2}+2 \nu_{\mathrm{Q}}^{2}}
$$

This is interpretable as meaning that the "bunching" instability will set in for a value of the wavenumber $\nu$ that is at most $1 / \sqrt{3}$ of the "quenching" limit $\nu_{\mathrm{Q}}$.

\section{ATTACHED MEMBRANE}

Up to this stage our qualitative considerations have been restricted to what occurs in the limit $\varepsilon \rightarrow 0$, which should be a good approximation in the higher temperature range, above the limit (41), where the symmetric embedded vortex defect is stable. However, since the pion mass $m_{\pi}$ given by Eq. (11) will actually not be small compared with $\eta$ but of the same order of magnitude, the effects of the symmetry breaking term proportional to $\varepsilon$ will be significant in the lower temperature range where the vortex defects will be of the asymmetric "superconducting" kind.

In the rest of this section we will assume that $\varepsilon>0$ but that the pion mass is small compared to the scale of symmetry breaking. In Sec. XIV we return to the question of what happens in the case of a realistic (i.e., large) pion mass.

The effect of $\varepsilon \neq 0$ is to break the degeneracy of the circular set of the equilibrium states that were characterized by $\chi^{*} \chi=0, \phi^{*} \phi=\eta_{\Theta}^{2}$, by adding unequal adjustment energy terms, $\delta_{+} V$ and $\delta_{-} V$ say, to the restricted local minima at $\sigma=\sigma_{+}$and $\sigma=\sigma_{-} \approx-\sigma_{+}$, thereby breaking the degeneracy. On the basis of Eq. (38) these adjustments will be given approximately by

$$
\delta_{ \pm} V \simeq-\lambda \varepsilon \sigma_{ \pm}
$$

so that $\delta_{+} V$ is negative whereas $\delta_{-} V$ will be positive. This means that there will now be an absolute minimum only where $\sigma=\sigma_{+}$. It follows that a circuit round the set of what were previously degenerate equilibrium states must now cross a finite energy barrier whose height, $\delta V_{\Theta}$ say, will be given by the difference between the new (absolute) minimum where $\sigma=\sigma_{+}$and the (restricted) maximum where $\sigma$ $=-\sigma_{-}$(in both cases for vanishing values of the pion components) which will be given by

$$
\delta V_{\Theta}=\delta_{-} V-\delta_{+} V
$$

On the basis of the estimates (9) this works out as

$$
\delta V_{\Theta} \simeq 2 \varepsilon \lambda \eta_{\Theta}
$$

as long as $\eta_{\Theta}^{3} \gg \varepsilon$, while near the critical temperature, i.e., when $\Theta \simeq \sqrt{2} \eta$, it would have an order of magnitude that is obtainable from Eq. (31) as

$$
\delta V_{\Theta} \approx 2 \varepsilon^{4 / 3} \lambda
$$

In the case of ordinary cosmic strings it is well known [28] that if the symmetry giving rise to the strings is weakly broken by an explicit symmetry breaking term, then the strings become boundaries of membrane-like defects. This membrane is the locus where the phase of the string order parameter changes by $2 \pi$ (if we consider circling the string in the transverse plane, then the change in phase is no longer uniform as it would be without symmetry breaking, but the phase change is localized along one ray in the transverse plane, i.e. on a membrane in three-dimensional space).

The presence of this energy barrier means that it will no longer be possible to have a strictly isolated exactly axisymmetric vortex defect, but that instead there will be composite defects (of a kind whose mechanics and classical [29] and quantum [30] decay processes have been considered in the context of axion theory) that cannot be isolated but must be attached to membrane defects (see Fig. 2 for a sketch). These membrane defects will not actually be "domain walls" in the strict sense (because the state outside will be the same on both sides) but they will have many of the same properties, including a thickness length scale $r_{\sigma}$ say, whose estimation (like that of $r_{\phi}$ ) can be obtained from the consideration that the energy will be minimized when the relevant gradient energy density, of the order of $\left(\sigma_{+} / r_{\sigma}\right)^{2}$, is comparable with the relevant barrier height $\delta V_{\Theta}$. Thus, as the analogue of Eq. (67) we obtain the general formula

$$
r_{\sigma}^{2} \approx \frac{\sigma_{+}^{2}}{\delta V_{\Theta}}
$$

The condition that the symmetry breaking coefficient $\varepsilon$ and hence also $\delta V_{\Theta}$ should be small evidently entails that $r_{\sigma}$ should be correspondingly large. It is only on scales small compared with this that the phase field distribution outside the string at a membrane boundary will retain the axially symmetric distribution assumed in Sec. IX. This radius will therefore act as the outer cutoff introduced in the equation of 


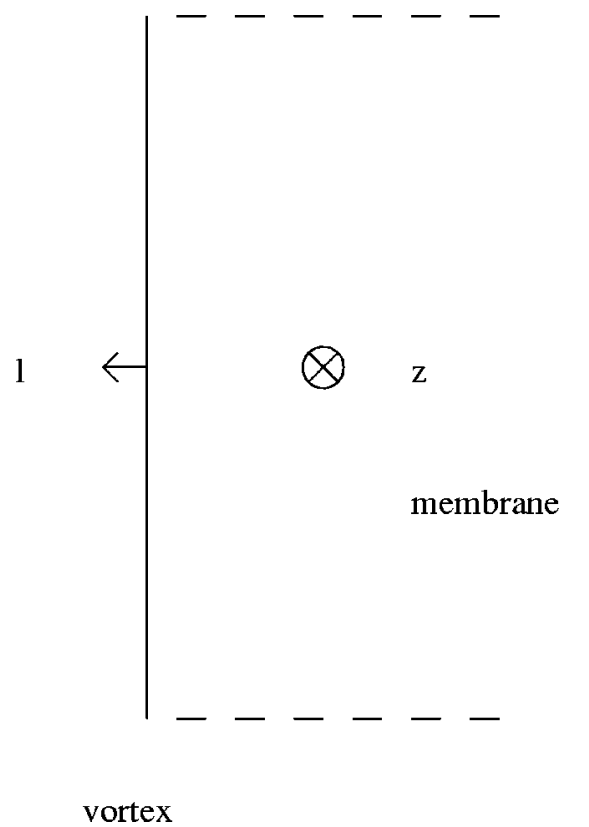

FIG. 2. Sketch of the vortex and the attached membrane. The vectors $l$ and $z$ in the figure correspond to the vectors $\lambda$ and $\zeta$ in the text in Sec. XII. The configuration is symmetric along the vertical axis, and the membrane is the surface to the right of the vortex (the solid line), as illustrated with the dashed lines.

state function (70). Thus when such symmetry breaking is present it will normally be appropriate to make the identification

$$
R_{\phi} \approx r_{\sigma}
$$

The membrane surface energy density resulting from the concentration of the phase gradient within the width $r_{\sigma}$ will be identifiable with the tension, $\mathcal{T}$ say, of the membrane, and will be given by the general formula

$$
\mathcal{T} \approx r_{\sigma} \delta V_{\Theta} .
$$

Although it will not be topologically stable such a membrane will be classically stabilized provided that after symmetry breaking, the value of the potential at the highest point in the original vacuum manifold (at $\sigma_{-}$) is lower than the potential at the origin. This classical stability criterion can be seen to be expressible as

$$
\delta_{-} V<\Delta V_{\Theta}^{[2]} .
$$

By rewriting Eq. (64) in the equivalent form

$$
\Delta V_{\Theta}^{[2]}=\frac{\lambda}{4} \eta_{\Theta}^{4}-\frac{\left(\nu_{\mathrm{Q}}^{2}-\nu^{2}\right)^{2}}{4 \lambda}
$$

it can be seen that this energy density must always satisfy the inequality

$$
\Delta V_{\Theta}^{[2]}<\frac{\lambda}{4} \eta_{\Theta}^{4}
$$

and hence that Eq. (84) imposes the requirement

$$
\eta_{\Theta}^{4}>\frac{4}{\lambda} \delta_{-} V .
$$

Evaluating the formula (77) for $\delta_{-} V$ using the first term in the estimate (30) for $\sigma_{-}$leads to the conclusion that classical stability of the membrane requires that $\Theta$ should be low enough for the inequality

$$
\eta_{\Theta}^{3} \geqslant 4 \varepsilon
$$

to be approximately satisfied. This conclusion justifies neglecting the second term in Eq. (30) and shows that it is not Eq. (31) but Eq. (30) that will be relevant as an order of magnitude estimate for $\sigma_{ \pm}$. We thereby conclude that

$$
\delta_{-} V \simeq \varepsilon \lambda \eta_{\Theta}
$$

which enables us to rewrite the classical stability criterion (84) in the form

$$
\left(\nu_{\mathrm{Q}}^{2}-\nu^{2}\right)^{2} \leqslant \lambda^{2} \eta_{\Theta}\left(\eta_{\Theta}^{3}-4 \varepsilon\right),
$$

from which the necessity of the condition (88) is obvious. The condition (88) also implies that the barrier height in the formula (81) will be given generally not by Eq. (80) but by Eq. (79), and hence by Eq. (33) that the membrane radius will be given by

$$
r_{\sigma} \approx \frac{1}{m_{\pi_{\Theta}}},
$$

with $m_{\pi_{\Theta}}$ as given by Eq. (34) rather than Eq. (35). Thus according to Eq. (83) the membrane tension $\mathcal{T}$ works out to be

$$
\mathcal{T} \approx \eta_{\Theta}^{2} m_{\pi_{\Theta}}
$$

or more explicitly

$$
\mathcal{T} \approx\left(1-\frac{\Theta^{2}}{2 \eta^{2}}\right)^{3 / 4} \eta^{2} m_{\pi} .
$$

\section{DRUM VORTON EQUILIBRIUM STATES}

We now examine the way in which the centrifugal effect of the string current in a closed loop can balance not just the contraction of the string tension, as in an ordinary vorton $[15,16,25]$, but also the contraction effect of the surface tension of the membrane that will be stretched across it in a drum type configuration of the kind whose investigation has been initiated more recently [31].

In so far as the sigma membrane is concerned, the general dynamical evolution can be described in terms of a unit vector, $\zeta^{\mu}$, orthogonal to the 3-dimensional sigma membrane world sheet, and on its boundary, another unit vector, $\lambda^{\mu}$, which is tangential to the world sheet but orthogonal to its boundary. In the two dimensional string world sheet that constitutes the boundary, there will be a preferred orthonormal diad of tangent vectors consisting of a preferred timelike unit vector $u^{\mu}$ and an orthogonal spacelike unit vector $\tilde{u}^{\mu}$, of 
which the latter is aligned with the current in the spacelike current configuration that we are considering. The set $\left\{u^{\mu}, \tilde{u}^{\mu}, \lambda^{\mu}, \zeta^{\mu}\right\}$ thus constitutes a complete orthonormal tetrad at any point on the bounding string (where it is physically well determined modulo sign reversals). In terms of such vectors the surface stress energy density tensor, $\mathcal{T}^{\mu \nu}$, of the membrane will be given by

$$
\mathcal{T}^{\mu \nu}=\mathcal{T}\left(\zeta^{\mu} \zeta^{\nu}-g^{\mu \nu}\right),
$$

where $\mathcal{T}$ is the fixed (background temperature dependent) membrane tension given by Eq. (92). The corresponding expression for the surface stress energy density tensor, $T^{\mu \nu}$, of the string at the boundary will be given in terms of a variable energy density, $U$, and a variable string tension, $T$, by

$$
T^{\mu \nu}=U u^{\mu} u^{\nu}-T \tilde{u}^{\mu} \tilde{u}^{\nu} .
$$

Systematically using curly script to distinguish quantities associated with the $2+1$ dimensional world sheet of the membrane from their analogues for the $1+1$ dimensional world sheet of the boundary string, the relevant dynamical equations will be succinctly expressible [25] in terms of a second fundamental tensor $K_{\mu \nu}^{\rho}$ of the string world sheet and of its analogue $\mathcal{K}_{\mu \nu}^{\rho}$ for the membrane. Since the latter evolves freely, its equation of motion will be of the simple general form

$$
\mathcal{T}^{\mu \nu} \mathcal{K}_{\mu \nu}^{\rho}=0 .
$$

The corresponding equation of motion for the string will have the non-homogeneous form

$$
T^{\mu \nu} K_{\mu \nu}^{\rho}=f^{\rho},
$$

with a force density on the right in which the dominant contribution (at least for configurations of large radius) will be produced by the attached membrane, whose effect will be given (subject to the orientation convention that the membrane tangent vector $\lambda^{\mu}$ at the boundary is outward directed) simply by

$$
f^{\rho}=\mathcal{T}^{\rho \nu} \lambda_{\nu} .
$$

In addition to this (in practise inwardly directed) membrane tension contribution, there will in principle be another (in practise outwardly) directed contribution arising from the magnetic field produced by the string current. However, as a result of the smallness of the electromagnetic coupling constant $e^{2} \simeq 1 / 137$, such a "magnetic spring" effect can be expected [32] to be relatively unimportant.

The simple isotropic form (94) of the membrane surface stress energy tensor allows us to evaluate the right hand side of Eq. (97) and the left hand side of Eq. (98) in more explicit form as

$$
f^{\mu}=-\lambda^{\mu}
$$

and

$$
\mathcal{T}^{\mu \nu} \mathcal{K}_{\mu \nu}^{\rho}=-\mathcal{T K}^{\rho}
$$

where $\mathcal{K}^{\mu}$ is the membrane curvature vector defined by

$$
\mathcal{K}^{\rho}=\mathcal{K}_{\nu}^{\nu \rho} .
$$

Since the world sheet of the membrane is just a hypersurface, with a uniquely (subject to a choice of orientation) defined normal $\zeta^{\mu}$, we can work in terms of its second fundamental form $\mathcal{K}_{\mu \nu}$ and the trace $\mathcal{K}$ as defined by

$$
\mathcal{K}_{\mu \nu}=\mathcal{K}_{\mu \nu}^{\rho} \zeta_{\rho}, \quad \mathcal{K}=\mathcal{K}_{\mu}^{\mu}=\mathcal{K}^{\rho} \zeta_{\rho},
$$

thus reducing the generic equation of free motion (96) for the membrane to the familiar more specialized form

$$
\mathcal{K}=0 .
$$

The membrane dynamical equation (103) will of course be trivially satisfied in the stationary, flat-drum- like configurations with which we are concerned here. The non-trivial part of the problem is the solution of Eq. (97) that governs the string boundary. Specifically our purpose is to look for vorton configurations that are characterized as being stationary with respect to a static background with respect to a timelike static symmetry generating vector $k^{\mu}$ that not only satisfies the Killing equation $k_{\nu ; \mu}+k_{; \mu \nu}=0$, but that is actually covariantly constant,

$$
k_{\mu ; \nu}=0 .
$$

The stationarity requirement imposes that this Killing vector be tangent to the world sheets of the membrane and of its string boundary. If we define (modulo another choice of sign) the spacelike unit string tangent vector $e^{\mu}$ to be orthogonal to $k^{\mu}$, the locally determined stress energy eigenvectors will be expressible in the form

$$
\begin{aligned}
& u^{\mu}=\left(1-v^{2}\right)^{-1 / 2}\left(k^{\mu}+v e^{\mu}\right), \\
& \tilde{u}^{\mu}=\left(1-v^{2}\right)^{-1 / 2}\left(e^{\mu}+v k^{\mu}\right),
\end{aligned}
$$

where $v$ is what will be interpretable as the rotation speed of the vorton, which will be given in terms of the phase frequency variables introduced in Eq. (48), as specified with respect to the vorton rest frame, by $v=\omega / k$.

The second fundamental tensor works out in this case to be given by an expression of the form

$$
K_{\mu \nu}^{\rho}=e_{\mu} e_{\nu} K^{\rho},
$$

in which the curvature trace vector

$$
K^{\rho}=K_{\nu}^{\nu \rho}
$$

will be given simply by

$$
K^{\rho}=e^{\nu} \nabla_{\nu} e^{\rho} .
$$

For a flat and circular configuration with radius $R$, the curvature vector can thus be seen to be given in terms of the radially outward directed unit normal $\lambda^{\rho}$ simply by 


$$
K^{\rho}=-\frac{1}{R} \lambda^{\rho} .
$$

By combining this with Eqs. (95) and (105) it can be seen that the left hand side of the string dynamical equation (97) will be given explicitly by

$$
T^{\mu \nu} K_{\mu \nu}^{\rho}=-\frac{U v^{2}-T}{\left(1-v^{2}\right) R} \lambda^{\rho} .
$$

Using this in conjunction with the expression (99) for the force density on the right, which is also proportional to $\lambda^{\rho}$, the dynamical equation (97) can be seen to reduce to the simple explicit form

$$
U v^{2}-T=\left(1-v^{2}\right) R \mathcal{T} .
$$

In this drum vorton equilibrium equation, it is to be recalled that $U$ is the string energy density (its locally preferred rest frame with relative motion $v$ ) and that $T$ is the corresponding (state dependent) string tension, while $\mathcal{T}$ is the (fixed) "drum" tension characterizing the membrane. Thus for an arbitrary string state characterized by a chosen energy density $U$ and an associated, necessarily smaller, value of the string tension, $T<U$, it will be possible to obtain a circular drum vorton solution with arbitrarily large radius $R$ by taking a correspondingly high (but always subluminal) rotation velocity value given by

$$
v^{2}=\frac{T+R \mathcal{T}}{U+R \mathcal{T}}
$$

\section{DEFECTS IN PION HADRODYNAMICS}

Since by Eq. (11) the symmetry breaking parameter $\varepsilon$ will be given in terms of the observable pion mass $m_{\pi}$ by

$$
\varepsilon \simeq \frac{\eta}{\lambda} m_{\pi}^{2}
$$

the minimal (necessary but not sufficient) defect stability requirement (88) can be expressed as the inequality

$$
m_{\pi}^{2} \leqslant \frac{\lambda \eta^{2}}{4}\left(1-\frac{\Theta^{2}}{2 \eta^{2}}\right)^{3 / 2} .
$$

As long as $\Theta$ is not too large compared with $\sqrt{2} \eta$, this will be compatible_-albeit rather marginally_with the observed ratio, $m_{\pi} / \eta \approx 2$, due to the fairly large value that is empirically [19] measured for the dimensionless constant $\lambda \approx 25$.

The foregoing requirement is obtained from the condition (90) in the limit for which the phase gradient magnitude $\nu$ has its maximum "quenching" value $\nu_{\mathrm{Q}}$ as given by Eq. (56), a value that will in practice be unattainable due to the "bunching" instability limit given by Eq. (76).

For lesser values of $\nu$, a necessary-and, in view of Eq. (88), approximately sufficient-condition for the classical stability condition (90) to be satisfied is obtainable, by taking its square root, in the form

$$
\nu_{\mathrm{Q}}^{2}-\nu^{2} \leqslant \lambda \eta_{\Theta}^{2}-2 \frac{\lambda \varepsilon}{\eta_{\Theta}} .
$$

Using the expression (56) for the "quenching" limit $\nu_{\mathrm{Q}}$ and the formula (33) for the effective mass of the uncharged pion, this condition can be rewritten as

$$
\nu^{2} \geqslant 2\left(m_{\pi_{\Theta}}^{2}-\pi e^{2} \frac{\Theta^{2}}{2}\right) .
$$

Since the final, charge dependent, term is negative, this requirement would be satisfied automatically if the symmetry breaking term $m_{\pi}^{2}$ were small enough to satisfy the condition

$$
m_{\pi_{\Theta}}^{2}<\pi e^{2} \frac{\Theta^{2}}{2},
$$

which can be written more explicitly as

$$
m_{\pi}^{2}<\pi e^{2} \Theta^{2} \sqrt{1-\frac{\Theta^{2}}{2 \eta^{2}}}
$$

In practice however, as a result of the small value of the electromagnetic coupling constant $e^{2} \simeq 1 / 137$, the relatively large value of the symmetry breaking parameter $m_{\pi} / \eta \approx 2$ ensures that in the hadrodynamic application the condition (117) will fail throughout the relevant temperature range $\Theta$ $\lesssim \eta$.

Even when the condition (118) does not hold, the string defect stability condition (116) might still be satisfied for sufficiently large value of the current. However, as well as the difficulty of reconciling such a current with the upper limit on $\nu$ imposed by the bunching stability condition (76), there is the consideration that stability of the membrane against spontaneous formation of string surrounded holes in the membrane requires an energy barrier against formation of even the least energetic kind of strings, namely those for which $\nu$ vanishes. This suggests that genuinely stable defect formation will be possible only when Eq. (118) is satisfied.

The foregoing reasoning effectively rules out the case of the hadrodynamic application that motivated this investigation, but it raises the question of whether the kind of defects we have been considering might occur in other applications, involving the same type of $\mathrm{O}(4)$ sigma model but with weaker symmetry breaking. In terms of a dimensionless mass parameter $\tilde{m}$ and a dimensionless temperature parameter $\theta$ defined by

$$
\tilde{m}=\frac{m_{\pi}}{\eta}, \quad \theta=\frac{\Theta}{\sqrt{2} \eta}
$$

the situation may be summed up in the statement that the defects will be viable if and only if the temperature is in the limited range for which an inequality of the form 


$$
f\{\theta\}>\tilde{m}^{2}
$$

is satisfied, where the function $f\{\theta\}$ simply vanishes when $\theta$ is greater than unity, and is specified for $\theta<1$ as follows, in a manner that depends on whether $\theta$ is greater or less that the value $\theta_{\mathrm{Q}}$ given by

$$
\theta_{\mathrm{Q}}^{2}=\left(1+\frac{2 \pi e^{2}}{\lambda}\right)^{-1 / 2} \simeq 1-\frac{\pi e^{2}}{\lambda},
$$

at which the quantity $\nu_{\mathrm{Q}}^{2}$ given by Eq. (56) vanishes. For the higher range (41) one has

$$
\theta \geqslant \theta_{\mathrm{Q}} \Rightarrow f\{\theta\}=\frac{\lambda}{4}\left(1-\theta^{2}\right)^{3 / 2},
$$

by Eq. (114), while in the lower range (43) one has

$$
\theta \leqslant \theta_{\mathrm{Q}} \Rightarrow f\{\theta\}=\pi e^{2} \frac{\theta^{2}}{2}\left(1-\theta^{2}\right)^{1 / 2},
$$

by Eq. (118).

Clearly the condition for defect formation will never be satisfied if $\tilde{m}^{2}$ exceeds the maximum value of $f$, meaning roughly if $\tilde{m}^{2}>e^{2}$. In the more interesting case of a cosmological scenario with

$$
\tilde{m}^{2} \leqslant e^{2}
$$

the conclusion to be drawn is that as the cosmological temperature $\Theta$ drops past a first critical value $\Theta_{c}$ corresponding to $\theta=\theta_{\mathrm{c}}$ with $\theta_{\mathrm{c}}$ given roughly by $\theta_{\mathrm{c}} \simeq 1$, in approximate accordance with Eq. (39), but with a small deviation given in order of magnitude by

$$
1-\theta_{\mathrm{c}}^{2} \approx\left(\frac{\tilde{m}^{2}}{\pi e^{2}}\right)^{2},
$$

the universe would enter a regime in which the condition (120) is satisfied, so that the defects, in the form of string bounded membranes, would condense out and evolve. The strings would be superconducting from the outset unless $\Theta_{c}>\Theta_{Q}$, which implies that the [compared to Eq. (124)] relatively severe restriction

$$
\tilde{m}^{2} \leqslant \frac{\left(\pi e^{2}\right)^{3 / 2}}{\sqrt{\lambda}}
$$

is satisfied, and even in this extreme case they would rapidly become superconducting as the temperature drops below the value given by Eq. (43) and enters the regime characterized by Eq. (123). As a result of the superconductivity, some of the defect structure could be provisionally preserved (by mechanisms similar to those that have been considered for other kinds of string defects [33]) in the form of drum vortons of the kind described in Sec. XII. However, after passing through another lower critical temperature and entering a regime characterized roughly by

$$
\theta^{2} \lesssim\left(\pi e^{2}\right)^{-1} \tilde{m}^{2}
$$

the condition (120) would cease to hold, so there would be another phase transition in which any surviving string bounded membranes-including the drum vortons-would be destroyed.

\section{CONCLUSIONS}

In this paper we have studied the stabilization mechanism for embedded defects [1], with particular emphasis on the application to the classical bosonic $\mathrm{O}(4)$ sigma model of hadrodynamics. ${ }^{2}$

We have seen that below the stabilization threshold for an embedded defect of the traditional kind (with symmetric core) there will still be stablized cosmic string defects, but of asymmetric vortex type. These defects will automatically be superconducting, and this provides them with an extra stabilization mechanism. These superconducting string defects are stable above a threshold temperature $\Theta_{d}$ set by the strength of the explicit symmetry breaking term in the potential, i.e. by the pion mass in the case of hadrodynamics. In the absence of explicit symmetry breaking the defects remain stable until the temperature of recombination, at which point our thermal analysis breaks down.

In the case of explicit symmetry breaking, the superconducting vortices become boundaries of a new type of membrane-like defects which we call drum vortons, across which the change in the phase of the string order parameter is localized, and whose tension is given by the symmetry breaking mass, the pion mass in the case of hadrodynamics. We have seen that drum vortons can be stabilized by rotation.

In the case of hadrodynamics, the pion mass is too large for the superconducting vortices and drum vortons studied here to be stable. This is due to the large value of the pion mass relative to the QCD symmetry breaking scale and due to the large value of the self-coupling constant $\lambda$ relative to the small value of the gauge coupling $e^{2}$. However, in many grand unified models, we expect $\lambda$ to be small and the explicit symmetry breaking terms to be absent. In this case, the embedded strings with asymmetric core studied in this paper and their drum vortons would be stable.

Thus, we have identified a new class of defects which could be of great cosmological importance in the early universe. They could be used for baryogenesis (see, e.g., [35]) or for the generation of primordial magnetic fields (see, e.g., [36]). There are also severe cosmological constraints on models which admit such defects, a topic which we will come back to in a subsequent publication [37].

\footnotetext{
${ }^{2}$ After completion of this manuscript a report [34] appeared which discusses the stabilization of certain unstable strings and textures by the cosmological expansion.
} 


\section{ACKNOWLEDGMENTS}

The authors wish to thank J. Blanco-Pillado and A. Zhitnitsky for discussions. B.C. and A.C.D. thank Brown University and R.B. and A.C.D. thank UBC for hospitality while this work was in progress. This work was supported in part by the ESF COSLAB program and by a Royal SocietyCNRS exchange grant (B.C., A.C.D.), by PPARC (A.C.D.), by the U.S. Department of Energy under Contract DE-FG0291ER40688, Task A (R.B.), and by an Accord between CNRS and Brown University (B.C., R.B.). We are grateful to Herb Fried for securing this Accord.

\section{APPENDIX A: MEAN SQUARE AMPLITUDE IN THERMAL DISTRIBUTION}

To derive the coefficient in the ubiquitously useful formula (18), it will suffice to consider the case of a single component field, with small amplitude $\delta \varphi$ say, which can be analyzed as a sum of contributions from plane waves with angular frequency $\omega$ in different directions. From any such plane wave contribution, the mean square field fluctuation amplitude will receive an infinitesimal contribution $d\left\langle(\delta \varphi)^{2}\right\rangle$ that will be related to the corresponding infinitesimal contribution $d \mathcal{E}$ to the energy density $\mathcal{E}$ by a proportionality formula that (subject to use of the standard field normalization convention as above) will have the simple form

$$
d \mathcal{E}=\omega^{2} d\left\langle(\delta \varphi)^{2}\right\rangle \text {. }
$$

In a thermal distribution with temperature $\Theta$, the energy density contribution corresponding to an infinitesimal angular frequency range $d \omega$ will be given (in our units, for which the speed of light $c$, the Boltzmann constant $k$, and the Dirac Planck constant $\hbar=h / 2 \pi$ are all set to unity, i.e. $c=k=\hbar$ $=1$ ) by the well-known Bose-Einstein gas formula

$$
d \mathcal{E}=\frac{\omega^{3} d \omega}{2 \pi^{2}\left(\mathrm{e}^{\omega / \Theta}-1\right)} .
$$

Combining this with Eq. (A1) and integrating with the substitution $u=\omega / \Theta$, we find that the total mean square fluctuation amplitude will be given by

$$
\left\langle(\delta \varphi)^{2}\right\rangle=\frac{\Theta^{2}}{2 \pi^{2}} \int_{0}^{\infty} \frac{u d u}{\mathrm{e}^{u}-1} .
$$

Since the integral involved is well known to be given as a Riemann zeta function by

$$
\int_{0}^{\infty} \frac{u d u}{\mathrm{e}^{u}-1}=\zeta\{2\}=\frac{\pi^{2}}{6},
$$

we immediately obtain the simple final formula

$$
\left\langle(\delta \varphi)^{2}\right\rangle=\frac{\Theta^{2}}{12},
$$

of which the required multicomponent generalization (18) is now an obvious corollary.

\section{APPENDIX B: VARIATIONAL FORMULATION}

The conducting string model set up in Secs. VIII and IX can easily be cast into variational form in terms of an action integral

$$
\mathcal{I}=\int \mathcal{L}|\gamma|^{1 / 2} d^{2} \sigma
$$

Which is taken over a the string world sheet with internal coordinates $\sigma^{a}(a=0,1)$ and corresponding induced metric $\gamma_{a b}$ for a suitably chosen Lagrangian density scalar $\mathcal{L}$. In particular, in terms of the phase scalar $\varphi$ used above and of an appropriately specified auxiliary scalar $\Phi$, this Lagrangian can be given the standard form

$$
\mathcal{L}=-\frac{1}{2} \Phi^{2} \varphi_{\mid a} \varphi^{\mid a}-V\{\Phi\}
$$

with $\varphi$ and $\Phi$ as independently variable 2-surface supported fields using the general prescription [26]

$$
V=\frac{1}{2}(U+T)
$$

with

$$
\varphi_{\mid a} \varphi^{\mid a}=\nu^{2}, \quad \kappa=\Phi^{2} .
$$

In the present application to the model characterized by the equation of state (70), this prescription simply gives

$$
\Phi^{2}=\frac{\nu_{\mathrm{Q}}^{2}-\nu^{2}}{\lambda\left(m_{\chi}^{2}+\nu^{2}\right)}
$$

and hence

$$
\nu^{2}=\frac{\nu_{\mathrm{Q}}^{2}-m_{\chi}^{2} \lambda \Phi^{2}}{1+\lambda \Phi^{2}},
$$

so it immediately follows from Eqs. (70) and (71) that the required potential function $V\{\Phi\}$ will be given by

$$
V=\frac{m_{\chi}^{2} \lambda \Phi^{2}-\nu_{\mathrm{Q}}^{2}}{m_{\chi}^{2}+\nu_{\mathrm{Q}}^{2}}+\frac{1}{2} \ln \left\{\frac{R_{\phi}^{2}\left(m_{\chi}^{2}+\nu_{\mathrm{Q}}^{2}\right)}{1+\lambda \Phi^{2}}\right\} .
$$

[1] M. Nagasawa and R.H. Brandenberger, Phys. Lett. B 467, 205 (1999).

[2] X. Zhang, T. Huang, and R.H. Brandenberger, Phys. Rev. D 58, 027702 (1998).
[3] I. Halperin and A. Zhitnitsky, Phys. Lett. B 440, 77 (1998).

[4] M.M. Forbes and A.R. Zhitnitsky, J. High Energy Phys. 10, 013 (2001).

[5] D.T. Son, M.A. Stephanov, and A.R. Zhitnitsky, Phys. Rev. 
Lett. 86, 3955 (2001).

[6] D.T. Son, hep-ph/0108260.

[7] D.B. Kaplan and S. Reddy, Phys. Rev. Lett. 88, 132302 (2002).

[8] M.M. Forbes and A.R. Zhitnitsky, Phys. Rev. D 65, 085009 (2002).

[9] A.P. Balachandran and S. Digal, Int. J. Mod. Phys. A 17, 1149 (2002).

[10] T. Vachaspati and A. Achucarro, Phys. Rev. D 44, 3067 (1991).

[11] A. Achucarro, K. Kuijken, L. Perivolaropoulos, and T. Vachaspati, Nucl. Phys. B388, 435 (1992).

[12] M. Barriola, T. Vachaspati, and M. Bucher, Phys. Rev. D 50, 2819 (1994).

[13] A. Achucarro and T. Vachaspati, Phys. Rep. 327, 347 (2000); 327, 427 (2000).

[14] E. Witten, Nucl. Phys. B249, 557 (1985).

[15] R.L. Davis and E.P. Shellard, Nucl. Phys. B323, 209 (1989).

[16] B. Carter and X. Martin, Ann. Phys. (N.Y.) 227, 151 (1993).

[17] J.S. Schwinger, Ann. Phys. (N.Y.) 2, 407 (1957).

[18] M. Gell-Mann and M. Levy, Nuovo Cimento 16, 705 (1960).

[19] J.D. Walecka, Theoretical Nuclear and Subnuclear Physics (Oxford University Press, Oxford, 1995).

[20] L. Dolan and R. Jackiw, Phys. Rev. D 9, 2904 (1974).

[21] M. Axenides and L. Perivolaropoulos, Phys. Rev. D 56, 1973 (1997).

[22] M. Axenides, L. Perivolaropoulos, and M. Trodden, Phys. Rev. D 58, 083505 (1998).
[23] M. Axenides, L. Perivolaropoulos and T.N. Tomaras, Phys. Rev. D 58, 103512 (1998).

[24] P. Peter, Phys. Rev. D 45, 1091 (1992).

[25] B. Carter, in Formation and Interactions of Topological Defects, edited by C. Davis and R. Brandenberger, NATO Advanced Study Institute, Series B: Physics, Vol. 349 (Plenum, New York, 1995), pp. 303-348, hep-th/9611054.

[26] B. Carter, Ann. Phys. (Leipzig) 9, 247 (2000).

[27] B. Carter and P. Peter, Phys. Rev. D 52, 1744 (1995).

[28] A. Vilenkin and E.P.S. Shellard, Cosmic Strings and Other Topological Defects (Cambridge University Press, Cambridge, England, 1994).

[29] M. Nagasawa and M. Kawasaki, Phys. Rev. D 50, 4821 (1994).

[30] J. Preskill and A. Vilenkin, Phys. Rev. D 47, 2324 (1993).

[31] R. Cordero and A. Queijeiro, "Superconducting hybrid extended objects," report, Escuela Superior de Fisica y Mathematica, IPN, Mexico.

[32] P. Peter, Phys. Lett. B 298, 60 (1993).

[33] R.H. Brandenberger, B. Carter, A.C. Davis, and M. Trodden, Phys. Rev. D 54, 6059 (1996).

[34] R.S. Ward, Class. Quantum Grav. 19, L17 (2002).

[35] A.C. Davis and W.B. Perkins, Phys. Lett. B 393, 46 (1997).

[36] R.H. Brandenberger and X. Zhang, Phys. Rev. D 59, 081301 (1999).

[37] R. Brandenberger, B. Carter, and A.-C. Davis, "Microwave background constraints on decaying defects," Phys. Lett. B (to be published), hep-ph/0202168. 